\title{
Empirical Findings on Ontology Metrics
}

\author{
D. Rodríguez ${ }^{a, b *}$ M.A. Sicilia ${ }^{a}$, E. García $^{a}$ \\ ${ }^{a}$ Department of Computer Science \\ University of Alcalá, Ctra. Barcelona, Km. 31.6 \\ 28871 Alcalá de Henares, Madrid, Spain \\ ${ }^{b}$ Department of Computing and Communication Technologies \\ Oxford Brookes University \\ Wheatley Campus, Oxford OX33 1HX, UK \\ \{daniel.rodriguezg, msicilia, elena.garciab\}@uah.es
}

\begin{abstract}
Ontologies are becoming the preferred way of representing, dealing and reasoning with large volumes of information in several domains. In consequence, the creation, evaluation and maintenance of ontologies has become an engineering process that needs to be managed and measured using sound and reliable methods. As part of any ontology engineering or revision process, metrics can play a role helping in identifying possible problems or incorrect use of ontology elements, along with providing a kind of quality assessment that complements reviews requiring expert inspection. However, in spite of the fact that there are a number of ontology metric proposals described in the literature, there is a lack of empirical studies that provide a basis for their interpretation. This paper reports a systematic exploration of existing ontology metrics from a large set of ontologies extracted from the Swoogle search engine. The Ontorank value used inside the Swoogle to rank search results is used as a contrast for some existing metrics. The results show that the existing proposed metrics evaluated in general do not seem to be indicators of that ranking, but they can be helpful to identify particular kinds of ontologies.
\end{abstract}

Keywords. Ontology, metrics, Swoogle, Ontorank

\footnotetext{
${ }^{*}$ Corresponding author
} 


\section{Introduction}

Ontologies [4] are explicit representations of domain concepts and their relationships. More formally, an ontology defines the conceptualisations of a problem domain and a set of constraints on how terms can be combined to model the domain, using a formal language. Ontologies are nowadays used for different purposes in different systems, and as a consequence, they have become artifacts in the development of software.

In the Software Engineering domain, metrics play an important role in design, development and identifying software defects of future maintenance problems. Like it happens in the software engineering tasks, generally the construction of ontologies follows an iterative and incremental approach and a number of metrics has been proposed to measure different aspects such as reliability, reusability, cohesion, etc. (see for example [14]). However, unlike it happens in software engineering domain, there is a lack of empirical studies that validate how the different metrics are capable of judging quality properties and their interpretation. Inquiry on the relation of quality indicators and metrics is in consequence an important open challenge for a more systematic approach to developing ontology-based software systems. The problem with ontology metrics is that ontologies are very heterogeneous in their structure, objectives and level of formality. In consequence, there is a need to carry out exploratory studies that could eventually result in insights about the metrics applicable to different kinds of ontologies and their interpretation for each of them.

This paper reports on on such an exploratory study, carried out by measuring a large set of OWL ontologies obtained from the Swoogle repository together with a measure of their popularity using Ontorank [2]. The study has been carried out by implementing and using an open source software framework for computing ontology metrics expressed in the Ontology Web Language $(\mathrm{OWL})^{1}$, that was already used in a previous preliminary study [9]. OWL has been chosen as the language is a $\mathrm{W} 3 \mathrm{C}$ recommendation and has rapidly become the standard language for expressing formal ontologies. The results of the study point out that current metrics are not directly interpretable in a general way, but there seems to be different categories of ontologies that could be discernible from the value of the metrics.

The rest of the paper is structured as follows. Section 2 covers the background on ontology metrics and some related work. Next, Section 3 describes the statistical analyses performed together with some findings.

\footnotetext{
${ }^{1}$ http://www.w3.org/TR/owl-guide/
} 
Finally, Section 4 concludes the paper and outlines future research work.

\section{Background}

Ontologies have been a resource in the field of artificial intelligence since the 70's. However since the inception of the Semantic Web, ontologies have become the principal recourse to integrate and deal with online information, and a new set of standards to formally represent them has been developed and gained widespread adoption. The Ontology Web Language (OWL) is one of such standards that belongs to a family of knowledge representation languages created for the Semantic Web [1]. The OWL has reached the status of W3C (World Wide Web Consortium) recommendation. From a technical point of view OWL extends the RDF (Resource Description Framework) and RDF-S (RDF Schema) allowing us to integrate a variety of applications using XML as interchange syntax.

The OWL has evolved since its inception in 2004 to a new specification in 2009 (OWL2) with different sub-languages depending on the expressiveness and reasoning capabilities provided. There are however common elements and OWL ontologies are composed of (i) classes that can be nested as sets of individuals, (ii) individuals as instances of classes, i.e., objects of the domain and (iii) properties as binary relations between individuals. It is also possible to specify property domains, cardinality ranges and reasoning on ontologies. From these elements a number of authors have proposed metrics to measure the quality of ontologies.

Several authors have proposed metrics related to ontologies or adapted from Object-oriented software. For example, Yao et al. [17] proposed a set on metrics to measure the cohesion in ontologies such as the number of root classes, number of leaf classes and average depth of inheritance tree of all leaf nodes. Authors validated the metrics both theoretically and empirically. Their empirical validation consisted of comparing statistically the results of the metrics and subjective evaluation of eighteen evaluators.

Tatir et al. [13] propose three orthogonal dimensions to evaluate the quality of an ontology. The first dimension is quality of the ontology schema representing the real world. The second dimension evaluates the content of the ontology, i.e, how well populated is the ontology and if it reflects the real world. Finally, the third dimension evaluates if the of instances and relations agree with the schema. Schema metrics proposed by the authors include relationship, attribute and inheritance richness. Instance metrics are divided into (i) knowledge base metrics such as class richness, average 
population and cohesion and (ii) class metrics which include importance, fullness, inheritance richness, relationship richness connectivity and readability. Authors analysed two general purpose ontologies SWETO and TAP as well as the GlycO (Glycomics Ontology) ontology.

Vrandečić and Sure [15] propose a framework for designing ontologies though a process of normalisation and considering stable metrics, i.e., metrics considering the open world assumption.

Metrics and metric frameworks can help with processes or methodologies for the design of ontologies or ontological tools. A well-known methodology for the evaluation of ontological adequacy of taxonomic relationships is OntoClean defined by Guarino and Welty [5]. Another process is the OntoMetric process defined by Lozano-Tello and Gómez-Pérez [7]. OntoMetric is a process to select ontologies using a broad range of metrics, not only those that can be collected form the actual ontologies.

A suite a of ontology metrics to measure design complexity has been recently proposed [18], reporting an analysis of desirable properties for metrics described by Weyuker, and providing results of an empirical evaluation in which the metrics proposed are shown to be discriminating ontologies that are considered by the authors to be of different complexity.

In another direction, Ding et al. [3, 2] have developed Swoogle, a semantic search engine in which the popularity of a semantic web document is defined using the Ontorank measure adapted from the well-known PageRank algorithm [12]. That ranking metric is an external criteria that can be used to contrast ontology metrics. The problem with the ranking is that its interpretation as a quality measure is not evident and has not been empirically studied. However, as other quality measures are not available for ontologies, it represents an interesting opportunity to contrast existing metrics.

We also developed an open source framework to extract the OWL ontology metrics called OntoMetrics. The OntoMetrics is an open source Java implementation that makes use the Java libraries of Protégé ${ }^{2}$ an ontology editor that provides an application programmers interface (API) for loading, saving and manipulating OWL and RDF files. In this paper we have use this framework to collect metrics from a large number of ontologies together with their popularity measured using Ontorank.

${ }^{2}$ http://protege.stanford.edu/ 


\section{Empirical Analysis of Ontology Data}

In this section, the metrics gathered are described and then the results of the statistical analysis is reported. Only basic metrics related to the main elements of ontologies have been implemented and used. Particularly, metrics dealing with classes inferred (i.e., defined classes) or axioms were not considered as a majority of the ontologies available for the study lacked these kinds of constructs.

\subsection{Dataset}

In order to empirically analyse the relationships between the previously defined metrics (Section 2), we downloaded around 1,500 ontologies defined in OWL using Swoogle ${ }^{3}$, which is a search engine for the Semantic Web. A crawler was developed ad hoc that submits a blank query to Swoogle and then navigates the pages downloading automatically the OWL files. Only ontologies expressed in OWL were considered as they represent the majority of the available ones, and allows for an homogeneous analysis.

Using the previously commented Ontometrics framework, we collected the following metrics:

- No. of Classes (noc) is a simple count of the number of classes $(|C|)$ contained in the ontology.

- No. of Instances (noi) is a simple count of the number of instances $(|I|)$ contained in the ontology.

- No. of Properties (nop) is a simple count of the number of properties $(|p|)$ contained in the ontology.

- No. of Root Classes metric (norc) [17] corresponds to the number of root classes (without superclasses) explicitly defined. Let $C$ the classes in an ontology: norc $=\left|C_{i}\right|, \neg \exists C_{j} \mid C_{i} \nsubseteq C_{j}$. The range of this metric is from 1 to $|C|$. There is at least one root and the larger the number of root classes, the more diverse the ontology will be.

- No. of Leaf Classes metric (nolc) [17] is the sum of all leaf classes, i.e., those without subclasses, in an ontology. Let $C$ the set of classes in an ontology: nolc $=\left|C_{i}\right|, \neg \exists C_{j} \mid C_{j} \not \subseteq C_{i}$. A a root class without out inheritance can also be considered a leaf class, the range of this metrics is from 1 to $|C|$.

\footnotetext{
${ }^{3}$ http://swoogle.umbc.edu/
} 
- Average Population metric (ap) [13] measures the average distribution of instances across all classes. Formally, it is defined as: $a p=\frac{|I|}{|C|}$ According the authors, this metric is supposed to be used in conjunction with the Class Richness metric as an indication if there is enough information in the ontology.

- Class Richness metric (cr) [13] is the ratio between the number of classes that have instances $\left(\left|C^{\prime}\right|\right)$ divided by the total number of classes. This metric provides an indication of how many instances are really related to classes defined in the schema.

- Explicit Depth of Subsumption Hierarchy (dosh). This metric corresponds to the maximum length of hierarchical relationships in the ontology explicitly defined, i.e., longer path from a root class.

- Relationship Richness metric ( $r r)$ [13] is defined as the ratio of the number of relationships divided by the sum of the number of subclasses plus the number of relationships: $r r=\frac{|P|}{|S C|+|P|}$, where $|P|$ is the number of relationships and $S C$ is the number of subclasses (or the number of inheritance relationships). According to the authors, this metric reflects the diversity of relations and placement of relations in the ontology. An ontology that contains many relationships other than class-subclass relations (values close to 1 ) is richer than a taxonomy with only class-subclass relationships (values close to zero).

- Inheritance Richness metric (ir) [13] is defined as the average number of subclasses per class. Formally, ir is defined as:

$$
i r=\frac{\sum_{C_{i} \in C}\left|H^{c}\left(C_{1}, C_{i}\right)\right|}{|C|}
$$

where $H^{c}\left(C_{1}, C_{i}\right) \mid$ is the number of subclasses $\left(C_{1}\right)$ for a class $\left(C_{i}\right)$ and the divisor $(C)$ is the total number of classes. According to the authors, this metric represents the distribution of information across different levels of the ontology inheritance tree and serves as an indicator of how well knowledge is grouped into different categories and subcategories in the ontology. Values close to zero indicate flat or horizontal ontologies representing perhaps more general knowledge while large values represent vertical ontologies describing detailed knowledge of a domain. 
- Ontorank [2, 3] is an adaptation from Google's PageRank [12] to emulate user's navigation behaviour at document level of granularity. OntoRank for a semantic document $a$ is defined as

$$
\operatorname{OntoRank}(a)=w P R(a)+\sum_{x \in O T C(a)} w P R(x)
$$

where $O T C(x)$ is the set of semantic documents that transitively $a$ imports or extends, and $w P R(a)$ is defined as: and

$$
\begin{array}{r}
w P R(a)=(1-d)+d \sum_{\operatorname{linkto}(x, a)} \frac{w P R(x) \cdot f(x, a)}{\sum_{\operatorname{link}(x,-, y)} f(x, y)} \\
f(x, a)=\sum_{\operatorname{link}(x, l, a)} \text { weight }(l)
\end{array}
$$

where $\operatorname{link}(a, l, b)$ represents a link from $a$ to $b$ semantic documents with semantic tag $l, \operatorname{linkto}(x, a)$ set of documents directly linked to $a$, weight $(l)$ is a user specified navigation preference on semantic links with tag $l, d$ is the probability of navigating from one document to another.

The Ontorank was directly provided by Swoogle, and represents an indicator of quality analogous to the PageRank [11] algorithm used in the Google search engine.

Table 1 summarizes the main metrics reported on the literature, classified by the kind of elements considered. Table 5 in Appendix A shows an excerpt from the data collected.

\subsection{Statistical Analysis}

As stated previously, we downloaded a large number of ontologies from Swoogle from which we could extract the metrics previously defined. The total number of ontologies from which we could collect all metrics was 1,413. Table 2 shows the descriptive statistics for the metrics collected.

The first interesting observation is that the average, median and third quartile values for most metrics are very low (see Table 2 and Figure 1), i.e., the vast majority of ontologies are small and quite flat (the ontology 
Table 1: Summary of Ontology Metrics used in this Work

\begin{tabular}{ll} 
Metric & Acronym \\
\hline \hline No. of Classes & noc \\
No. of Instances & noi \\
No. of Properties & nop \\
No. of Root Classes & norc \\
Number of Leaf Classes & nolc \\
Average Population & ap \\
Class Richness & $\mathrm{cr}$ \\
Explicit Depth of Subsumption Hierarchy & $\mathrm{dosh}$ \\
Inheritance Richness & $\mathrm{ir}$ \\
Relationship Richness Metric & $\mathrm{rr}$ \\
Ontorank & or \\
\hline
\end{tabular}

Table 2: Descriptive Statistics

\begin{tabular}{r|rrrrrrrrrr}
\hline & Avg & Med & Var & StdDev & Min & Max & Range & Q3 & StdSkw & StdKrt \\
\hline \hline or & 2.74 & 0.85 & 968.5 & 31.12 & 0.64 & 962.31 & 961.67 & 1 & 379 & 5300.1 \\
ap & 1.34 & 1 & 8.83 & 2.97 & 0 & 67 & 67 & 1.20 & 170.44 & 1469.95 \\
cr & 0.54 & 0.75 & 0.20 & 0.44 & 0 & 1 & 1 & 1 & -1.84 & -13.9 \\
dosh & 2.54 & 1 & 5.75 & 2.4 & 1 & 30 & 29 & 3 & 40.13 & 113.57 \\
ir & 0.34 & 0 & 0.17 & 0.42 & 0 & 1.83 & 1.83 & 0.75 & 9.97 & -9.31 \\
noc & 36.11 & 6 & 6166.3 & 78.53 & 1 & 945 & 944 & 34 & 80.44 & 293.25 \\
noi & 28.13 & 6 & 9522.8 & 97.59 & 0 & 991 & 991 & 9 & 94.85 & 328.43 \\
nolc & 27.46 & 5 & 3815.8 & 61.77 & 0 & 823 & 823 & 27 & 90.87 & 367.80 \\
nop & 24 & 0 & 2997.8 & 54.75 & 0 & 952 & 952 & 23 & 107.11 & 690.53 \\
norc & 6.69 & 5 & 127.37 & 11.29 & 0 & 194 & 194 & 6 & 131.7 & 857.18 \\
rr & 2.78 & 1 & 13.57 & 3.68 & 0 & 71 & 71 & 5 & 156.8 & 1326.26 \\
\hline
\end{tabular}




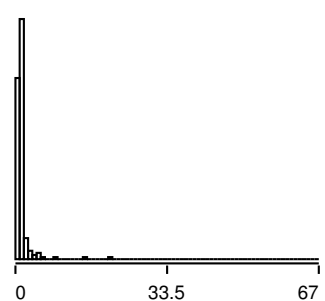

$a p$

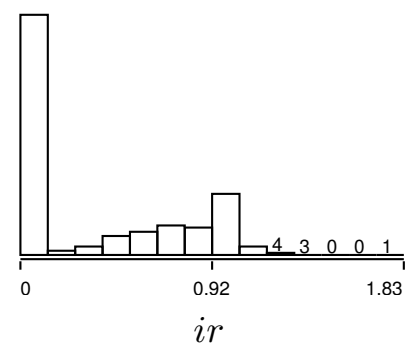

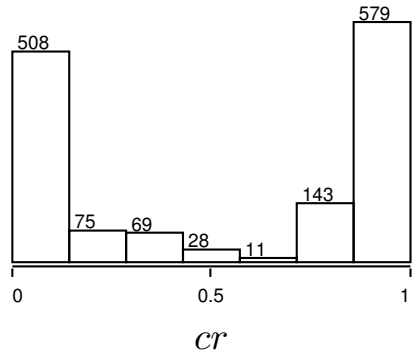

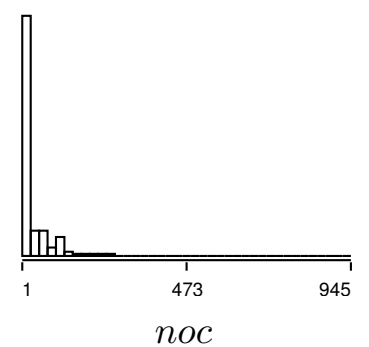

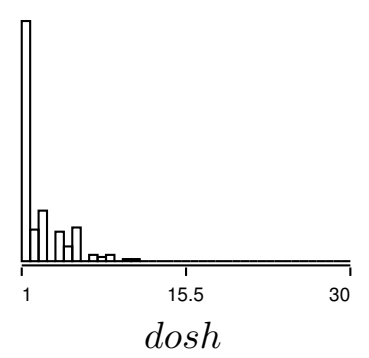

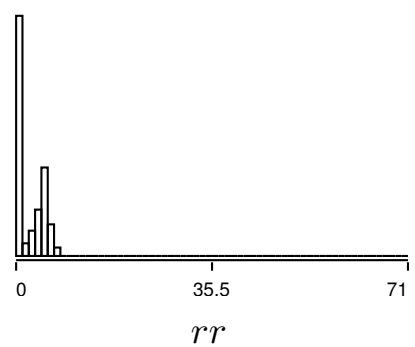

Figure 1: Histograms

hierarchy is in average very small). An exception is the ClassRichness (cr) metric with a ' $u$ ' shape which may be a reflection of two different ways of designing or using an ontology, either the instances are kept separately of the classes that define them or both classes and instances are stored together with the ontology.

Also in general, the distributions of the metrics are not following any identifiable distribution. Values of skewness and kurtosis in Table 2 allow us to discard normal distributions for all of them.

The few large values are statistically outliers as shown in Figure 2 by using the red colour. When analysing actual ontologies with large values, these correspond to special ontologies such as those in the Open Biomedical Ontologies (OBO) repository. For example, the NCI Thesaurus (ncithesaurus.owl) and the disease_ontology.owl are both extremely large ontologies with a special purpose. There are reasons for this that could be hypothesized to come from some differences on the languages and approaches to engineer ontologies in the biomedical domain [6].

Table 3 shows the Spearman rank correlation coefficient between the different metrics (also graphically in Figure 3). It can be observed that the OntoRank metric is not correlated with any other metric, and therefore, it seems that the popularity of an ontology cannot be identified by the set of metrics used in this study. We have analysed this relationship with 


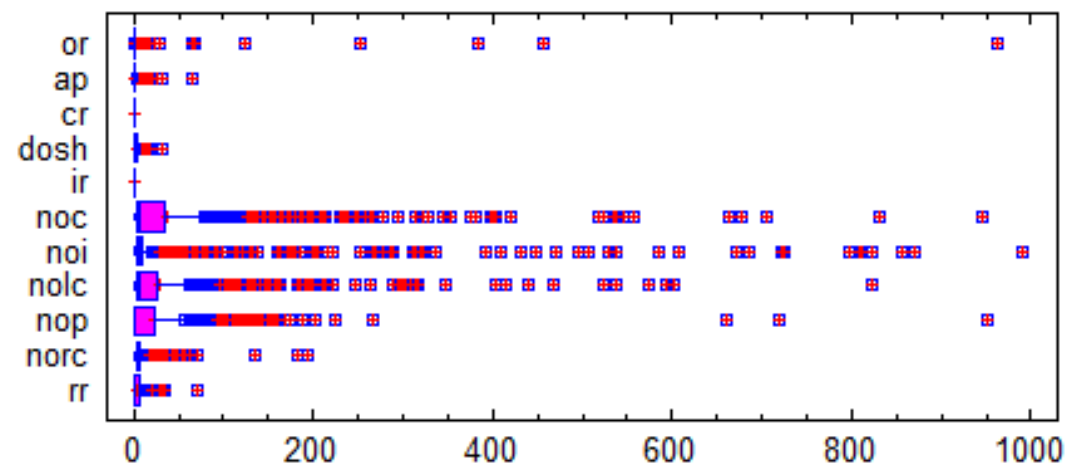

Figure 2: Box-and-whisker Plots

Table 3: Spearman Rank Correlation Coefficients

\begin{tabular}{|c|c|c|c|c|c|c|c|c|c|}
\hline & $\mathrm{cr}$ & dosh & $i r$ & $n o c$ & noi & nolc & nop & norc & $r r$ \\
\hline$a p$ & 0.6878 & -0.4387 & -0.4735 & -0.3157 & 0.6261 & -0.2800 & -0.2754 & 0.1959 & 0.4837 \\
\hline $\mathrm{cr}$ & & -0.6995 & -0.7151 & -0.5607 & 0.2789 & -0.5105 & -0.6500 & 0.0787 & 0.7250 \\
\hline dosh & & & 0.9776 & 0.8073 & 0.1370 & 0.7460 & 0.7959 & 0.0998 & -0.7443 \\
\hline$i r$ & & & & 0.7853 & 0.0848 & 0.7148 & 0.7709 & 0.0288 & -0.7526 \\
\hline$n o c$ & & & & & 0.3173 & 0.9812 & 0.6583 & 0.4275 & -0.4580 \\
\hline$n o i$ & & & & & & 0.3369 & 0.2525 & 0.5574 & 0.1828 \\
\hline nolc & & & & & & & 0.6154 & 0.4613 & -0.3822 \\
\hline nop & & & & & & & & 0.2487 & -0.5948 \\
\hline norc & & & & & & & & & 0.2400 \\
\hline
\end{tabular}

other data mining approaches using several Weka's [16] classifiers such as regression or classification trees to confirm this effect.

The relationship between the rest of the metrics were contrasted using factor analysis. Table 4 shows the resulting factor loadings using principal component analysis with standarised values as an extraction method in which 3 components were extracted.

The first component is characterized by high values in dosh, ir, noc that roughly measure depth and breadth of the subsumption hierarchy, i.e., metrics related with the schema of the ontology. The second component is characterized by a high correlation with the ap and noi so it is more related with the content of the ontology rather than the schema. and properties and also with relationship richness that relates both of them. In the third component, the largest values are also related to the ap and noi.

There are some threats to the validity of this study. First of all, we analysed single files, but Semantic Web documents can import or extend other documents in order to be able of working together. Coupling [10] or cohesion [8] metrics should be also be included. Furthermore, the metrics 


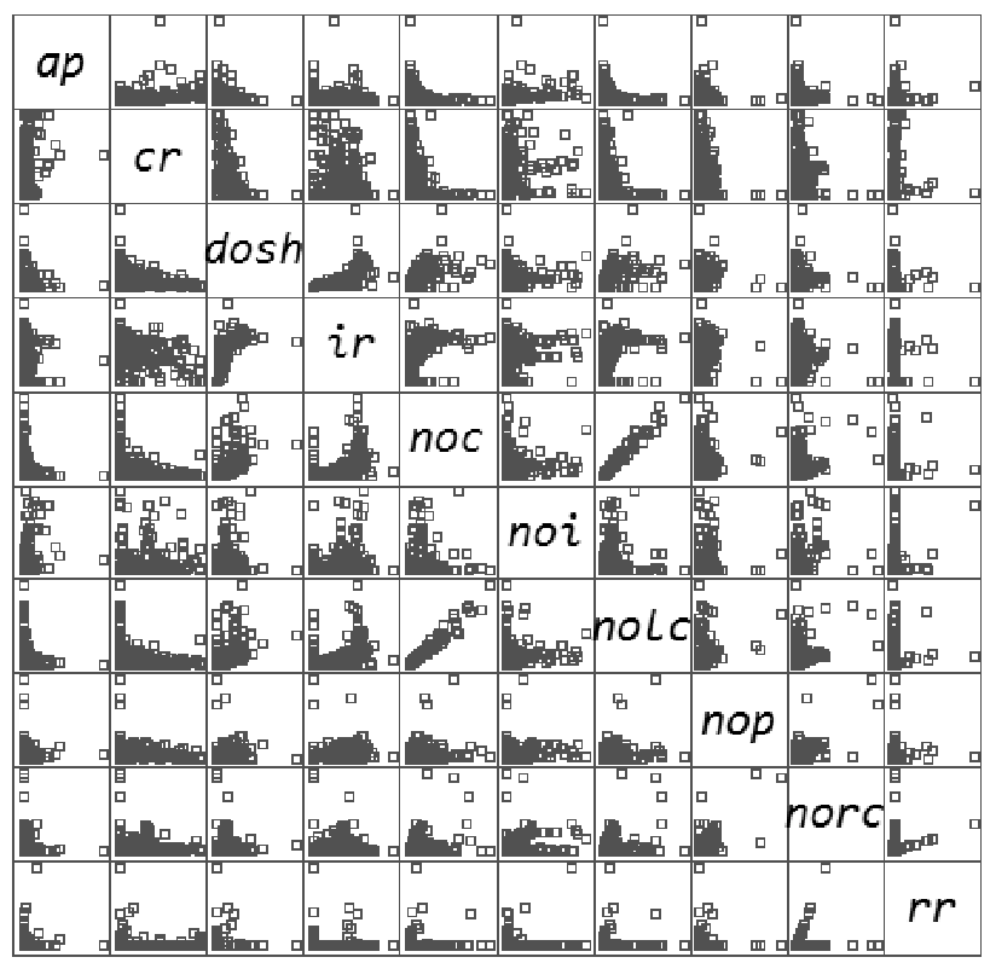

Figure 3: Graphical representation of the Spearman Rank Correlations

Table 4: Factorial Analysis of the Ontological Metrics

\begin{tabular}{c|lll}
\hline & Factor 1 & Factor 2 & Factor 3 \\
\hline \hline ap & -0.0786494 & 0.551809 & -0.681197 \\
cr & -0.727543 & 0.33865 & 0.167987 \\
dosh & 0.855587 & -0.205325 & -0.164372 \\
ir & 0.825037 & -0.29855 & -0.300246 \\
noc & 0.835051 & 0.129089 & 0.290564 \\
noi & 0.344157 & 0.653781 & -0.458953 \\
nolc & 0.797677 & 0.165676 & 0.337331 \\
nop & 0.676127 & 0.259735 & 0.0893104 \\
norc & 0.437119 & 0.61869 & 0.338713 \\
rr & -0.351663 & 0.566569 & 0.2992 \\
\hline
\end{tabular}


computed were extracted from the OWL files, with no associated reasoning process. This entails that the metrics do not consider elements that would be potentially inferred from reasoners, e.g. automatic classifications or subsumption relationships that are not explicit. While this may seem a major limitation, the fact that most ontologies do not contain axioms or defined classes (classes expressed through logical necessary and sufficient conditions) suggests that explicit elements are enough for the empirical assessment of metrics in the current state of development of ontologies.

\section{Conclusions and Future Work}

This paper addressed the empirical analysis of a large number of ontologies through a collection of metrics in order to propose interpretations for the metrics that can be subject to hypothesis contrast and eventually lead to a practical applicability of these metrics. The OntoRank was used as a contrast as the only available assessment mechanism that can be automatically computed without requiring expert assessment of the ontologies. It should be noted that such kind of indicators cannot substitute but complement expert of logical assessments as those that have been already reported in the literature.

To do so, we developed an open source tool, called Ontometrics, used to collect the set of metrics presented in this paper together with their Ontoranking value provided by Swoogle. The results showed that there is no correlation between the popularity of an ontology and the metrics used in this work. There are some peculiarities for example most ontologies are quite flat and small, perhaps reflecting that most of the ontologies are not mature. Some exceptions can be found in the OBO foundry ${ }^{4}$ repository which contains mature ontologies from the biomedical domain. In this case, although it is difficult to establish concrete thresholds to define when an ontology needs to be improved, such metrics helped in identifying ontologies for special purposes such as thesaurus.

Future work will be directed to further develop our metrics framework with new metrics and update the framework to the new OWL API ${ }^{5}$. There is also room for further analysis of the metrics, for example, analysing how ontologies evolve by carrying out longitudinal studies accounting for the evolution of ontologies.

\footnotetext{
${ }^{4}$ http://www .obofoundry.org/

${ }^{5}$ http://owlapi.sourceforge.net/
} 


\section{References}

[1] Tim Berners-Lee, James Hendler, and Ora Lassila. The semantic web. Scientific American, 2001.

[2] Li Ding, T. Finin, A. Joshi, Yun Peng, Rong Pan, and P. Reddivari. Search on the semantic web. Computer, 38(10):62-69, Oct. 2005.

[3] Li Ding, Tim Finin, Anupam Joshi, Rong Pan, R. Scott Cost, Yun Peng, Pavan Reddivari, Vishal Doshi, and Joel Sachs. Swoogle: a search and metadata engine for the semantic web. In Proceedings of the thirteenth ACM international conference on Information and knowledge management (CIKM'04), pages 652-659, New York, NY, USA, 2004. ACM.

[4] Thomas R. Gruber. Toward principles for the design of ontologies used for knowledge sharing. International Journal of Human-Computer Studies, 43(5-6):907-928, 1995.

[5] Nicola Guarino and Christopher Welty. Evaluating ontological decisions with ontoclean. Communications of the ACM, 45(2):61-65, 2002.

[6] Robert Hoehndorf, Anika Oellrich, Michel Dumontier, Janet Kelso, Dietrich Rebholz-Schuhmann, and Heinrich Herre. Relations as patterns: bridging the gap between OBO and OWL. BMC Bioinformatics, 11:441, 2010.

[7] A. Lozano-Tello and A. Gómez-Pérez. ONTOMETRIC: A method to choose the appropriate ontology. Journal of Database Management, 15(2):1-18, 2004.

[8] Yinglong Ma, Beihong Jin, and Yulin Feng. Semantic oriented ontology cohesion metrics for ontology-based systems. Journal of Systems and Software, 83(1):143-152, 2010.

[9] N. Manouselis, M.A. Sicilia, and D. Rodríguez. Exploring ontology metrics in the biomedical domain. In Workshop on Knowledge Representation and Decision Making (KREAM), International Conference in Computational Science (ICCS), pages 2313-2322. Elsevier, May 2010.

[10] A.M. Orme, H. Tao, and L.H. Etzkorn. Coupling metrics for ontologybased system. IEEE Software, 23(2):102-108, mar. 2006.

[11] Larry Page, Sergey Brin, R. Motwani, and T. Winograd. The pagerank citation ranking: Bringing order to the web, 1998. 
[12] Lawrence Page, Sergey Brin, Rajeev Motwani, and Terry Winograd. The pagerank citation ranking: Bringing order to the web. Technical Report 1999-66, Stanford InfoLab, November 1999.

[13] Samir Tartir, I. Budak Arpinar, Michael Moore, Amit P. Sheth, and Boanerges Aleman-Meza. Ontoqa: Metric-based ontology quality analysis. In IEEE Workshop on Knowledge Acquisition from Distributed, Autonomous, Semantically Heterogeneous Data and Knowledge Sources, 2005.

[14] Mike Uschold and Michael Grninger. Ontologies: principles, methods, and applications. Knowledge Engineering Review, 11(2):93-155, 1996.

[15] Denny Vrandečić and York Sure. How to design better ontology metrics. In Enrico Franconi, Michael Kifer, and Wolfgang May, editors, The Semantic Web: Research and Applications, volume 4519 of Lecture Notes in Computer Science, pages 311-325. Springer Berlin/Heidelberg, 2007.

[16] I.H. Witten and E. Frank. Data Mining: Practical machine learning tools and techniques. Morgan Kaufmann, San Francisco, 2005.

[17] Haining Yao, Anthony Mark Orme, and Letha Etzkorn. Cohesion metrics for ontology design and application. Journal of Computer Science, 1(1):107-113, 2005.

[18] Hongyu Zhang, Yuan-Fang Li, and Hee Beng Kuan Tan. Measuring design complexity of semantic web ontologies. Journal of Systems and Software, 83(5):803-814, 2010.

\section{A Data Collected Example}




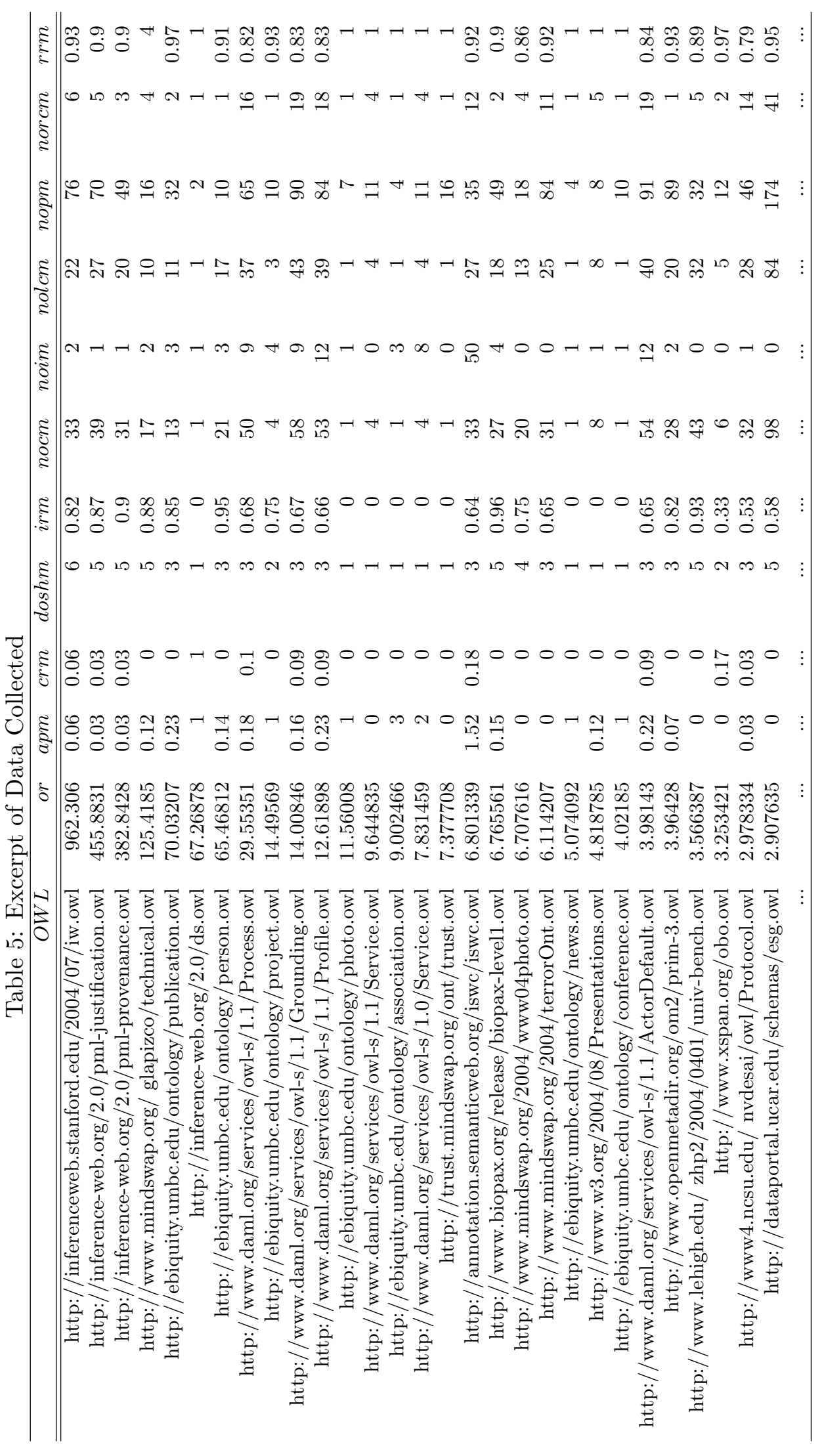

